\title{
Using the Weibull accelerated failure time regression model to predict time to health events
}

\author{
Enwu $\operatorname{Liu}^{1 *}, 2$, Karen $\operatorname{Lim}^{1}$
}

1 Musculoskeletal Health and Ageing Research Program, Mary MacKillop Institute for Health Research, Australian Catholic University, Melbourne, Victoria, Australia 2 College of Medicine and Public Health, Flinders University, Adelaide, South Australia, Australia

* enwu.liu@acu.edu.au

\begin{abstract}
We describe a statistical method protocol to use a Weibull accelerated failure time (AFT) model to predict time to a health-related event. This prediction method is quite common in engineering reliability research but rarely used for medical predictions such as survival time. A worked example for how to perform the prediction using a published dataset is included.
\end{abstract}

\section{Keywords}

Weibull regression, prediction, survivial time

\section{Introduction}

In medical literature, most prediction models are used to predict the probability of an event occurring or condition developing over a specified time period, such as the Framingham 10 Year Risk of General Cardiovascular Disease [1] and FRAX: a Tool for Estimating 10 year Fracture Risk. [2] In engineering reliability research, it is common to use Weibull accelerated failure time (AFT) models to predict 'time to failure', for example the lifespan of a machine; when a component will need replacement; and an optimal maintenance schedule that maximizes reliability of the entire system. [3] Weibull AFT models are also used to predict shelf-life of perishable foods and warranty period of goods. $[4,5]$ This statistical method estimates when the event will occur without being bound to a defined time period (i.e. absolute time; when a component will need replacement vs. relative time; 10-y risk of needing replacement). In addition to predicting engineering or mechanic events, this statistical method might also be useful for predicting medical events such as fracture, myocardial infarction and death.

In this paper, we have not intended to develop and present a prediction tool. Rather, we aim to illustrate how to use the Weibull AFT model and assess point accuracy in a medical context.

\section{Weibull distribution}

The Weibull distribution is also called the type III extreme value distribution. [6] The distribution has three parameters: the location parameter $\mu$, the scale parameter $\rho$ and 
the shape parameter $\gamma$. The location parameter $\mu$ is predetermined as the minimum value in the distribution. In survival or failure analysis, $\mu$ of 0 usually selected to produce a two-parameter distribution.

The cumulative distribution function (CDF) for a two-parameter Weibull distributed random variable $T \sim W(\rho, \gamma)$ is given as

$$
F_{T}(t ; \rho, \gamma)=1-\exp \left[-\left(\frac{t}{\rho}\right)^{\gamma}\right]
$$

for $t \geq 0, \rho>0, \gamma>0$ and $F(t ; \rho, \gamma)=0$ for $t<0$

The probability density function $(\mathrm{PDF})$ of the Weibull distribution is given as

$$
f_{T}(t ; \rho, \gamma)=F^{\prime}(t ; \rho, \gamma)=\frac{\gamma}{\rho}\left(\frac{t}{\rho}\right)^{\gamma-1} \exp \left[-\left(\frac{t}{\rho}\right)^{\gamma}\right]
$$

The survival function of the Weibull distribution is given as

$$
S_{T}(t)=1-F_{T}(t)=\exp \left[-\left(\frac{t}{\rho}\right)^{\gamma}\right]
$$

The mean survival time or mean time to failure (MTTF) is given as

$$
\begin{array}{rlr}
E(T) & =\int_{0}^{\infty} S(t) d t \quad \text { (Darth Vader rule) } \\
& =\int_{0}^{\infty} \exp \left[-\left(\frac{t}{\rho}\right)^{\gamma}\right] d t & \text { let }\left(\frac{t}{\rho}\right)^{\gamma}=u \Rightarrow t=\rho u^{\frac{1}{\gamma}} \\
& =\int_{0}^{\infty} e^{-u} \rho \frac{1}{\gamma} u^{\frac{1}{\gamma}-1} d u & \\
& =\rho \frac{1}{\gamma} \Gamma\left(\frac{1}{\gamma}\right) & \text { note } \frac{1}{\gamma} \Gamma\left(\frac{1}{\gamma}\right)=\Gamma\left(\frac{1}{\gamma}+1\right) \\
& =\rho \Gamma\left(\frac{1}{\gamma}+1\right) &
\end{array}
$$

\section{Log-Weibull distribution}

The log-Weibull distribution is also called Gumbel distribution, or type I extreme value 30 distribution. [7] Let $T \sim W(\rho, \gamma)$ and $Y=g(T)=\log T$ is a one-one transformation from support $\mathcal{T}=\{t \mid t>0\}$ to $\mathcal{Y}=\{y \mid-\infty<y<\infty\}$. The inverse of $Y$ is

$$
T=g^{-1}(Y)=e^{Y}
$$

and the Jacobian is given as

$$
|J|=\left|\frac{d g^{-1}(Y)}{d Y}\right|=e^{Y}
$$


The probability density function of $Y$ is then derived from (2)

$$
\begin{aligned}
f_{Y}(y) & =f_{T}\left(g^{-1}(y)\right)|J|=\frac{\gamma}{\rho}\left(\frac{e^{y}}{\rho}\right)^{\gamma-1} \exp \left[-\left(\frac{e^{y}}{\rho}\right)^{\gamma}\right] e^{y}=\frac{\gamma}{\rho} \frac{e^{y(\gamma-1)}}{\rho^{(\gamma-1)}} e^{y} \exp \left[-\frac{e^{\gamma y}}{\rho^{\gamma}}\right] \\
& =\gamma \frac{e^{\gamma y}}{\rho^{\gamma}} \exp \left[-\frac{e^{\gamma y}}{\rho^{\gamma}}\right] \quad \operatorname{note}\left(\rho^{\gamma}=e^{\gamma \log \rho}\right) \\
& =\gamma \frac{e^{\gamma y}}{e^{\gamma l o g \rho}} \exp \left[-\frac{e^{\gamma y}}{e^{\gamma l o g} \rho}\right]=\gamma e^{\gamma y-\gamma \log \rho} \exp \left[-e^{\gamma y-\gamma \log \rho}\right] \\
& =\gamma e^{\gamma(y-\log \rho)} \exp \left[-e^{\gamma(y-\log \rho)}\right] \quad\left(\operatorname{let} \gamma=\frac{1}{b} \text { and } \log \rho=a\right) \\
& =\frac{1}{b} \exp \left(\frac{y-a}{b}\right) \exp \left[-\exp \left(\frac{y-a}{b}\right)\right] \text { where }-\infty<y<\infty
\end{aligned}
$$

It shows $\log$-Weibull distribution has a Gumbel distribution $G(a, b)$, where $a=\log \rho$ and $b=\frac{1}{\gamma}$. The CDF of the log-Weibull distribution can be derived from the above PDF or by the definition directly.

$$
F_{Y}(y)=P(Y \leq y)=P(\log (T) \leq y)=P\left(T \leq e^{y}\right)=F_{T}\left(e^{y}\right)
$$

By $\mathrm{Eq}(1)$ we get

$$
\begin{aligned}
F_{Y}(y) & =F_{T}\left(e^{y}\right)=1-\exp \left[-\left(\frac{e^{y}}{a}\right)^{\gamma}\right]=1-\exp \left[-\frac{e^{\gamma y}}{a^{\gamma}}\right] \\
& =1-\exp \left[-\frac{e^{\gamma y}}{e^{\gamma \log \rho}}\right]=1-\exp \left[-e^{\gamma(y-\log \rho)}\right] \\
& =1-\exp \left[-\exp \left(\frac{y-a}{b}\right)\right] \text { where } \gamma=\frac{1}{b}, \log \rho=a
\end{aligned}
$$

The survival function of $Y=\log (T)$ is given by

$$
S_{Y}(y)=1-F_{Y}(y)=\exp \left[-\exp \left(\frac{y-a}{b}\right)\right]
$$

The hazard function is given by

$$
h_{Y}(y)=\frac{f_{Y}(y)}{S_{Y}(y)}=\frac{1}{b} \exp \left(\frac{y-a}{b}\right)
$$

\section{Weibull AFT regression model}

Let $T$ be the survival time. Suppose we have a random sample of size $n$ from a target population. For a subject $i(i=1,2, \ldots, n)$, we have observed values of covariates

$x_{i 1}, x_{i 2}, \ldots, x_{i p}$ and possibly censored survival time $t_{i}$. We can express the Weibull AFT model as

$$
\log \left(t_{i}\right)=\beta_{0}+\beta_{1} x_{i 1}+\ldots+\beta_{p} x_{i p}+\sigma \epsilon_{i}=\boldsymbol{x}_{\boldsymbol{i}}^{\prime} \boldsymbol{\beta}+\sigma \epsilon_{i}, \quad i=1,2, \ldots, n
$$

where $\boldsymbol{\beta}=\left(\beta_{0}, \ldots, \beta_{p}\right)$ are the regression coefficients of interest, $\sigma$ is a scale parameter and $\epsilon_{1}, \ldots \epsilon_{n}$ are independent and identically distributed according to a Gumbel distribution with PDF

$$
f_{\epsilon}(x)=\exp (x) \exp [-\exp (x)]
$$

and the $\mathrm{CDF}$

$$
F_{\epsilon}(x)=1-\exp [-\exp (x)]
$$


Note $\mathrm{Eq}(9)$ and $\mathrm{Eq}(10)$ are equal to set $a=0, b=1$ in $\mathrm{Eq}(5)$ and $\mathrm{Eq}(6)$. We denote it as a $G(0,1)$ distribution or a standard Gumbel distribution.

Now, let us find the PDF of $T$ from $\mathrm{Eq}(8)$

$$
\begin{aligned}
& \log (T)=\boldsymbol{x}^{\prime} \boldsymbol{\beta}+\sigma \epsilon \\
& \Rightarrow T=e^{\boldsymbol{x}^{\prime} \boldsymbol{\beta}+\sigma \epsilon} \\
& \Rightarrow \epsilon=g^{-1}(T)=\frac{\log (T)-\boldsymbol{x}^{\prime} \boldsymbol{\beta}}{\sigma} \\
& \Rightarrow|J|=\left|\frac{d\left(g^{-1}(T)\right)}{d T}\right|=\frac{1}{\sigma T}
\end{aligned}
$$

Puting $\mathrm{Eq}(11)$ and $\mathrm{Eq}(12)$ to $\mathrm{Eq}(9)$ we get

$$
\begin{aligned}
f_{T}(t) & =f_{\epsilon}\left(g^{-1}(t)\right)|J|=\exp \left(\frac{\log (t)-\boldsymbol{x}^{\prime} \boldsymbol{\beta}}{\sigma}\right) \exp \left[-\exp \left(\frac{\log (t)-\boldsymbol{x}^{\prime} \boldsymbol{\beta}}{\sigma}\right)\right] \frac{1}{\sigma t} \\
& =\left(\frac{t}{\exp \left(\boldsymbol{x}^{\prime} \boldsymbol{\beta}\right)}\right)^{\frac{1}{\sigma}} \exp \left[-\left(\frac{t}{\exp \left(\boldsymbol{x}^{\prime} \boldsymbol{\beta}\right)}\right)^{\frac{1}{\sigma}}\right] \frac{1}{\sigma t} \\
& =\frac{1 / \sigma}{\exp \left(\boldsymbol{x}^{\prime} \boldsymbol{\beta}\right)}\left(\frac{t}{\exp \left(\boldsymbol{x}^{\prime} \boldsymbol{\beta}\right)}\right)^{\frac{1}{\sigma}-1} \exp \left[-\left(\frac{t}{\exp \left(\boldsymbol{x}^{\prime} \boldsymbol{\beta}\right)}\right)^{\frac{1}{\sigma}}\right]
\end{aligned}
$$

Comparing $\operatorname{Eq}(13)$ with $\mathrm{Eq}(2)$ and let $\gamma=\frac{1}{\sigma}$ and $\rho=\exp \left(\boldsymbol{x}^{\prime} \boldsymbol{\beta}\right)$, we can see $T$ has a Weibull distribution $T \sim W\left(\exp \left(\boldsymbol{x}^{\prime} \boldsymbol{\beta}\right), \frac{1}{\sigma}\right)$.

Referring to $\mathrm{Eq}(3)$, now the survival function of $T \sim W\left(\exp \left(\boldsymbol{x}^{\prime} \boldsymbol{\beta}\right), \frac{1}{\sigma}\right)$ can be written as

$$
S_{T}(t)=\exp \left[-\left(\frac{t}{\exp \left(\boldsymbol{x}^{\prime} \boldsymbol{\beta}\right)}\right)^{\frac{1}{\sigma}}\right]
$$

Referring to $\operatorname{Eq}(4)$ the expected survival time of $W\left(\exp \left(\boldsymbol{x}^{\prime} \boldsymbol{\beta}\right), \frac{1}{\sigma}\right)$ is given as

$$
E(T)=\exp \left(\boldsymbol{x}^{\prime} \boldsymbol{\beta}\right) \Gamma(\sigma+1)
$$

Since most statistical software use $\log (T)$ to calculate these parameters, let us show the distribution and characteristics of the $\log (T)$. Let

$$
\begin{gathered}
Y=\log (T)=\boldsymbol{x}^{\prime} \boldsymbol{\beta}+\sigma \epsilon \\
\Rightarrow \epsilon=g^{-1}(Y)=\frac{Y-\boldsymbol{x}^{\prime} \boldsymbol{\beta}}{\sigma} \\
\Rightarrow|J|=\left|\frac{d\left(g^{-1}(Y)\right)}{d Y}\right|=\frac{1}{\sigma}
\end{gathered}
$$

Putting $\mathrm{Eq}(16)$ and $\mathrm{Eq}(17)$ to $\mathrm{Eq}(9)$, we get

$$
f_{Y}(y)=f_{\epsilon}\left(g^{-1}(Y)\right)|J|=\frac{1}{\sigma} \exp \left(\frac{y-\boldsymbol{x}^{\prime} \boldsymbol{\beta}}{\sigma}\right) \exp \left[-\exp \left(\frac{y-\boldsymbol{x}^{\prime} \boldsymbol{\beta}}{\sigma}\right)\right]
$$

Comparing $\operatorname{Eq}(18)$ to $\operatorname{Eq}(5)$ we can see that $Y$ i.e. $\log (T)$ has a $G\left(\boldsymbol{x}^{\prime} \boldsymbol{\beta}, \sigma\right)$ distribution. We also can see the error term $\epsilon$, which has a $G(0,1)$ distribution. similar to the error term in a simple linear regression with a $N\left(0, \sigma^{2}\right)$ distribution.

Referring to $\mathrm{Eq}(13)$ and $\mathrm{Eq}(18)$, we can see in the Weibull AFT model, $T$ has a Weibull $W\left(\exp \left(\boldsymbol{x}^{\prime} \boldsymbol{\beta}, \frac{1}{\sigma}\right)\right)$ distribution, and $\log (T)$ has a Gumbel $G\left(\boldsymbol{x}^{\prime} \boldsymbol{\beta}, \sigma\right)$ distribution.

From Eq (7) the survival function of $Y$ i.e. $\log (T)$ is given as

$$
S_{Y}(y)=\exp \left[-\exp \left(\frac{y-\mathbf{x}^{\prime} \beta}{\sigma}\right)\right]
$$


And the expectation of $Y$ i.e $\log (T)$ is calculated as

$$
\begin{aligned}
E(Y)= & E(\log (T))=\int_{-\infty}^{\infty} y f_{Y}(y) d y \\
= & \int_{-\infty}^{\infty} y \frac{1}{\sigma} \exp \left(\frac{y-\boldsymbol{x}^{\prime} \boldsymbol{\beta}}{\sigma}\right) \exp \left[-\exp \left(\frac{y-\boldsymbol{x}^{\prime} \boldsymbol{\beta}}{\sigma}\right)\right] d y \quad\left(\text { let } z=\exp \left(\frac{y-\boldsymbol{x}^{\prime} \boldsymbol{\beta}}{\sigma}\right)\right) \\
= & \frac{1}{\sigma} \int_{0}^{\infty}\left(\sigma \log z+\boldsymbol{x}^{\prime} \boldsymbol{\beta}\right) z \exp (-z) d\left(\sigma \log z+\boldsymbol{x}^{\prime} \boldsymbol{\beta}\right) \quad\left(y=\sigma \log z+\boldsymbol{x}^{\prime} \boldsymbol{\beta}\right) \\
= & \int_{0}^{\infty}\left(\sigma \log z+\boldsymbol{x}^{\prime} \boldsymbol{\beta}\right) \exp (-z) d z=\sigma \int_{0}^{\infty} \log z \exp (-z) d z+\boldsymbol{x}^{\prime} \boldsymbol{\beta} \int_{0}^{\infty} \exp (-z) d z \\
= & \sigma \int_{0}^{\infty} \frac{\partial}{\partial \alpha}\left[z^{\alpha} e^{-z}\right]_{\alpha=0} d z+\boldsymbol{x}^{\prime} \boldsymbol{\beta} \\
= & \sigma \frac{\partial}{\partial \alpha}\left[\int_{0}^{\infty} z^{\alpha} e^{-z} d z\right]_{\alpha=0}\left(\text { differentiating under the integral, note } \frac{d z^{\alpha}}{d \alpha}=\log (z) z^{\alpha}\right) \\
& +\boldsymbol{x}^{\prime} \boldsymbol{\beta} \\
= & \sigma \Gamma(1)^{\prime}+\boldsymbol{x}^{\prime} \boldsymbol{\beta} \\
= & \boldsymbol{x}^{\prime} \boldsymbol{\beta}-\sigma \xi \quad\left(\text { where } \Gamma(1)^{\prime}=-\xi \approx-0 \cdot 57721, \quad \xi \text { is the Euler-Mascheroni Constant }\right)
\end{aligned}
$$

Note, $\operatorname{since} \log (x)$ is a concave down function by Jensen's inequality,

$E(\log (T)) \leq \log (E(T)), \mathrm{Eq}(15)$ is the correct formula to calculate the expected survival time rather than $\exp \left(\boldsymbol{x}^{\prime} \boldsymbol{\beta}-\sigma \xi\right)$.

\section{Parameter estimation for the Weibull AFT model}

The parameters of Weibull AFT model can be estimated by the maximum likelihood method. The likelihood function of the $n$ observed $\log (t)$ time, $y_{1}, y_{2}, \ldots, y_{n}$ is given by

$$
\begin{aligned}
L\left(\beta, \sigma ; y_{i}\right) & =\prod_{i=1}^{n}\left[f_{Y}\left(y_{i}\right)\right]^{\delta_{i}}\left[S_{Y}\left(y_{i}\right)\right]^{1-\delta_{i}} \\
& =\prod_{i=1}^{n}\left\{\frac{1}{\sigma} \exp \left(\frac{y_{i}-\boldsymbol{x}^{\prime} \boldsymbol{\beta}}{\sigma}\right) \exp \left[-\exp \left(\frac{y_{i}-\boldsymbol{x}^{\prime} \boldsymbol{\beta}}{\sigma}\right)\right]\right\}\left\{\exp \left[-\exp \left(\frac{y_{i}-\boldsymbol{x}^{\prime} \boldsymbol{\beta}}{\sigma}\right)\right]\right\}
\end{aligned}
$$

where $\delta_{i}$ is the event indicator for ith subject with $\delta_{i}=1$ if an event has occurred and $\delta_{i}=0$ if the event has not occurred. The maximum likelihood estimates $p+1$ parameters $\sigma, \beta_{1} \ldots, \beta_{p}$. We can take the $\log$ of the likelihood function and use the Newton-Raphson method to calculate these parameters. Most statistical software packages can perform these calculations.

\section{Calculate expected survival time using the Weibull AFT model}

In reliability research, expected survival time is called mean time to failure (MTTF) or mean time between failures (MTBF). [8]

Suppose we want to predict a person $i$ 's mean survival time $t_{i}$ using the Weibull AFT model. First, we use the maximum likelihood estimation (MLE) method of $\mathrm{Eq}(20)$ to calculate the $\hat{\boldsymbol{\beta}}$ and $\hat{\sigma}$ then by the invariance property of MLE, we use Eq(15) to calculate the predicted MTTF directly.

$$
t_{i}=E\left(t_{i}\right)=\exp \left(\boldsymbol{x}_{\boldsymbol{i}}^{\prime} \hat{\boldsymbol{\beta}}\right) \Gamma(\hat{\sigma}+1)
$$


After we calculate the MTTF, we can use the Delta method to calculate the confidence 79 interval for the MTTF. We will treat the predicted MTTF as a function of $\hat{\boldsymbol{\beta}}$ and $\hat{\sigma}$. The standard error of the MTTF can be calculated as

$$
S E=\left\{\left(\begin{array}{c}
\frac{\partial E\left(\hat{t}_{i}\right)}{\partial \hat{\boldsymbol{\beta}}} \\
\frac{\partial E\left(t_{i}\right)}{\partial \hat{\sigma}}
\end{array}\right)^{t} \Sigma_{\hat{\sigma} \hat{\boldsymbol{\beta}}}\left(\begin{array}{c}
\frac{\partial E\left(\hat{t}_{i}\right)}{\partial \hat{\boldsymbol{\beta}}} \\
\frac{\partial E\left(t_{i}\right)}{\partial \hat{\sigma}}
\end{array}\right)\right\}^{\frac{1}{2}}
$$

where $\Sigma_{\hat{\sigma} \hat{\boldsymbol{\beta}}}$ is the variance-covariance matrix of $\hat{\boldsymbol{\beta}}$ and $\hat{\sigma}$. The variance-covariance matrix can be estimated by the observed Fisher information of the Weibull AFT model. The (1- $\alpha) \%$ confidence interval is given as

$$
\hat{t_{i}}-z_{1-\frac{\alpha}{2}} S E<t_{i}<\hat{t}_{i}+z_{1-\frac{\alpha}{2}} S E
$$

where $\alpha$ is the type I error and $z$ is the quantile of the standard normal distribution.

\section{Calculate median survival time using Weibull AFT model}

Another important statistic in survival analysis is the median survival time or percentile survival time. The $p$ th percentile of survival time is calculated from the survival function. For the individual $i$ the $p$ th percentile of survival time is calculated as

$$
S_{T}\left(t_{i}(p)\right)=\frac{100-p}{100}
$$

For the Weibull AFT model, we use $\mathrm{Eq}(14)$ to calculate $p$ th survival time of an individual $i$.

$$
\begin{aligned}
& S_{T}\left(t_{i}\right)=\exp \left[-\left(\frac{t_{i}}{\exp \left(\boldsymbol{x}^{\prime} \boldsymbol{\beta}\right)}\right)^{\frac{1}{\sigma}}\right]=\frac{100-p}{100} \\
& \Rightarrow\left(\frac{t_{i}}{\exp \left(\boldsymbol{x}^{\prime} \boldsymbol{\beta}\right)}\right)^{\frac{1}{\sigma}}=-\log \left(\frac{100-p}{100}\right) \\
& \Rightarrow t_{i}=\left[-\log \left(\frac{100-p}{100}\right)\right]^{\sigma} \exp \left(\boldsymbol{x}_{\boldsymbol{i}}^{\prime} \boldsymbol{\beta}\right)
\end{aligned}
$$

After we obtain $\hat{\boldsymbol{\beta}}$ and $\hat{\sigma}$ from $\mathrm{Eq}(20)$ and use the the invariance property of MLE, the median survival time is estimated by

$$
t_{i}(50)=(\log 2)^{\hat{\sigma}} \exp \left(\boldsymbol{x}_{\boldsymbol{i}}^{\prime} \hat{\boldsymbol{\beta}}\right)
$$

We can treat the estimated survival time percentile as a function of $\hat{\sigma}$ and $\hat{\boldsymbol{\beta}}$ when $p$ is fixed by using the Delta method to calculate the standard error of predicted $p$ th survival time. The method is the same as in $\mathrm{Eq}(22)$ and $\mathrm{Eq}(23)$

\section{Minimum prediction error survival time (MPET)}

Both mean and median survival time estimates are biased when the sample size is small and the model includes censoring. To attenuate bias, Henderson et al. purposed a method to find the optimum prediction time with the minimum prediction error. [9] They specified that if an observed survival time $t$ falls in the interval $\frac{p}{k}<t<k p$ where 
$p$ is the predicted survival time and $k>1$ then the prediction is accurate. The probability of prediction error $E_{k}$ condition on the predicted time $p$ is given as

$$
\begin{aligned}
P\left(E_{k} \mid p\right) & =P(T<p / k)+P(T>k p) \quad \text { (observed time falls outside the bounds) } \\
& =1-S_{T}(p / k)+S_{T}(k p)
\end{aligned}
$$

The minimum probability of prediction error $P\left(E_{k} \mid p\right)$ is achieved when

$$
f_{T}(p / k)=k^{2} f(k p)
$$

Now let us calculate the minimum prediction error for the Weibull AFT model. From $\mathrm{Eq}(13)$ we get

$$
\begin{aligned}
& f_{T}(p / k)=\frac{1 / \sigma}{\exp \left(\boldsymbol{x}^{\prime} \boldsymbol{\beta}\right)}\left(\frac{p / k}{\exp \left(\boldsymbol{x}^{\prime} \boldsymbol{\beta}\right)}\right)^{\frac{1}{\sigma}-1} \exp \left[-\left(\frac{p / k}{\exp \left(\boldsymbol{x}^{\prime} \boldsymbol{\beta}\right)}\right)^{\frac{1}{\sigma}}\right] \\
& k^{2} f_{T}(k p)=k^{2} \frac{1 / \sigma}{\exp \left(\boldsymbol{x}^{\prime} \boldsymbol{\beta}\right)}\left(\frac{k p}{\exp \left(\boldsymbol{x}^{\prime} \boldsymbol{\beta}\right)}\right)^{\frac{1}{\sigma}-1} \exp \left[-\left(\frac{k p}{\exp \left(\boldsymbol{x}^{\prime} \boldsymbol{\beta}\right)}\right)^{\frac{1}{\sigma}}\right]
\end{aligned}
$$

Applying $\mathrm{Eq}(25)$ and canceling the common parts, we get

$$
k^{1-\frac{1}{\sigma}} \exp \left[-\left(\frac{p / k}{\exp \left(\boldsymbol{x}^{\prime} \boldsymbol{\beta}\right)}\right)^{\frac{1}{\sigma}}\right]=k^{1+\frac{1}{\sigma}} \exp \left[-\left(\frac{k p}{\exp \left(\boldsymbol{x}^{\prime} \boldsymbol{\beta}\right)}\right)^{\frac{1}{\sigma}}\right]
$$

Taking the $\log$ of both sides we get

$$
\left(1-\frac{1}{\sigma}\right) \log (k)-\left(\frac{p / k}{\exp \left(\boldsymbol{x}^{\prime} \boldsymbol{\beta}\right)}\right)^{\frac{1}{\sigma}}=\left(1+\frac{1}{\sigma}\right) \log (k)-\left(\frac{k p}{\exp \left(\boldsymbol{x}^{\prime} \boldsymbol{\beta}\right)}\right)^{\frac{1}{\sigma}}
$$

Finally,when rearranging these terms we get

$$
p=\left[\frac{\frac{2}{\sigma} \log (k)}{k^{\frac{1}{\sigma}}-k^{-\frac{1}{\sigma}}}\right]^{\sigma} \exp \left(\boldsymbol{x}^{\prime} \boldsymbol{\beta}\right)
$$

Here $p$ is the minimum prediction error survival time. We may use the Delta method to get the standard error of the minimum prediction error survival time. Bootstrap methods also could be used to get a confidence interval.

\section{An example to predict the survival time}

\begin{tabular}{|c|c|c|c|c|c|}
\hline \multicolumn{6}{|c|}{ ID stage time age diagyr death } \\
\hline 1 & 1 & 0.6 & 77 & 76 & 1 \\
\hline 2 & 1 & 1.3 & 53 & 71 & \\
\hline 3 & 1 & 2.4 & 45 & 71 & \\
\hline 4 & 1 & 2.5 & 57 & 78 & \\
\hline 5 & 1 & 3.2 & 58 & 74 & \\
\hline & & & & & \\
\hline 46 & 2 & 6.2 & 74 & 72 & \\
\hline
\end{tabular}

We use a published larynx cancer dataset of 90 male participants [10] to demonstrate the calculations of the predictions. The dataset includes five variables: stage of cancer ('stage'; 1 =stage 1,2=stage 2,3=stage 3,4=stage 4); time to death or on-study time in months ('time'); age at diagnosis of larynx cancer ('age'); year of diagnosis ('diagyr'); and death indicator ('delta'; $0=$ alive, $1=$ dead). The authors added a unique particiapnt identifier variable (ID) to the dataset and renamed the variable 'delta' to 'death'. The dataset can be downloaded from here. The resulting dataset has the following structure 


\begin{tabular}{|c|c|c|c|c|}
\hline 47 & 2 & 7.0 & 62 & 73 \\
\hline 48 & 2 & 7.5 & 50 & 73 \\
\hline 49 & 2 & 7.6 & 53 & 73 \\
\hline 50 & 2 & 9.3 & 61 & 71 \\
\hline 51 & 3 & 0.3 & 49 & 72 \\
\hline 52 & 3 & 0.3 & 71 & 76 \\
\hline & & & & \\
\hline 39 & 4 & 3.8 & 84 & 74 \\
\hline 90 & 4 & 4.3 & 48 & 76 \\
\hline
\end{tabular}

We will use two predictors to predict the survival time: 1) stage of cancer and 2) age at cancer diagnosis. Since stage of cancer is a categorical variable, we will create three dummy variables for 'stage' and use stage 1 as the default reference group. The Weibull AFT model can be written as

$$
\log (T)=\beta_{0}+\beta_{1} * \text { stage } 2+\beta_{2} * \text { stage } 3+\beta_{3} * \text { stage } 4+\beta_{4} * \text { age }+\sigma \epsilon \quad \epsilon \sim G(0,1)
$$

Most statistical software can run the Weibull AFT regression model. Here we display R ${ }_{128}$ syntax

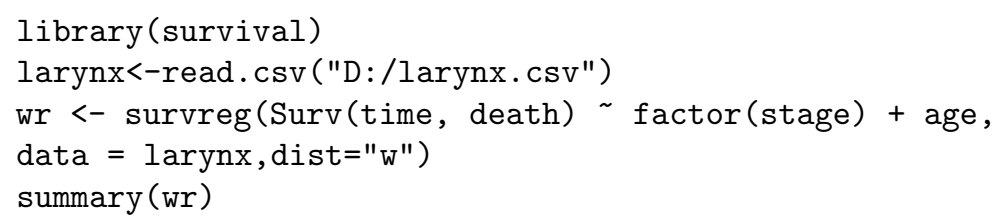

The above syntax yields the following results

Call:

survreg (formula $=\operatorname{Surv}($ time, death $) \sim$ factor (stage $)+$ age, data $=$ larynx, dist $=" w ")$

\begin{tabular}{|c|c|c|c|c|}
\hline & Value & Std. Error & $z$ & $\mathrm{p}$ \\
\hline (Intercept) & 3.5288 & 0.9041 & 3.903 & $9.50 e-05$ \\
\hline factor (stage) 2 & -0.1477 & 0.4076 & -0.362 & $7.17 e-01$ \\
\hline factor (stage) 3 & -0.5866 & 0.3199 & -1.833 & $6.68 e-02$ \\
\hline factor (stage) 4 & -1.5441 & 0.3633 & -4.251 & $2.13 e-05$ \\
\hline age & -0.0175 & 0.0128 & -1.367 & $1.72 \mathrm{e}-01$ \\
\hline $\log ($ scale) & -0.1223 & 0.1225 & -0.999 & $3.18 e-01$ \\
\hline \multicolumn{5}{|l|}{ Scale $=0.885$} \\
\hline \multicolumn{5}{|c|}{ Weibull distribution } \\
\hline \multicolumn{5}{|c|}{ Loglik $($ model $)=-141.4$} \\
\hline \multicolumn{5}{|c|}{ Chisq $=19.37$ on 4 degrees of freedom, $p=0.00066$} \\
\hline
\end{tabular}

Suppose we wanted to apply all three methods (MTTF, median and MPET) to predict the survival time of patient $\mathrm{ID}=46$, who had stage 2 cancer and was aged 74 years.

We would use $\operatorname{Eq}(21)$ to calculate his expected survival time (mean or MTTF)

$$
\begin{aligned}
M T T F_{46} & =E\left(\hat{t}_{46}\right)=\exp \left(\boldsymbol{x}_{\boldsymbol{i}}^{\prime} \hat{\boldsymbol{\beta}}\right) \Gamma(\hat{\sigma}+1) \\
& =\exp (3.5288-0.1477 * 1-0.5866 * 0-1.5441 * 0-0.0175 * 74) * \Gamma(1.885) \\
& =7.7 \text { (months })
\end{aligned}
$$

We would use $\mathrm{Eq}(24)$ to predict his median survival time

$$
\begin{aligned}
\text { Median }_{46} & =(\log 2)^{\hat{\sigma}} \exp \left(\boldsymbol{x}_{\boldsymbol{i}}^{\prime} \hat{\boldsymbol{\beta}}\right) \\
& =\log (2)^{0.885} \exp (3.5288-0.1477 * 1-0.5866 * 0-1.5441 * 0-0.0175 * 74) \\
& =5.8(\text { months })
\end{aligned}
$$


We would use $\mathrm{Eq}(26)$ to calculate the MPET and we fix $k=2$.

$$
\begin{aligned}
\operatorname{MPET}_{46} & =\left[\frac{\frac{2}{\sigma} \log (k)}{k^{\frac{1}{\sigma}}-k^{-\frac{1}{\sigma}}}\right]^{\sigma} \exp \left(\boldsymbol{x}^{\prime} \boldsymbol{\beta}\right) \\
& =\left[\frac{\frac{2}{\sigma} \log (k)}{2^{\frac{1}{0.885}-k^{-\frac{1}{0.885}}}}\right]^{0.885} * \exp (3.5288-0.1477 * 1-0.5866 * 0-1.5441 * 0-0.0175 * 74) \\
& =7.4 \text { (months) }
\end{aligned}
$$

It seems the three prediction methods MTTF, median and MPET produce estimates close to the actual survival time of patient $I D=46$, which was 6.2 months.

Note in R build in predict function for Weibull AFT model type $=$ "response" calculates $\exp \left(\boldsymbol{x}^{\prime} \hat{\boldsymbol{\beta}}\right)$ without the $\Gamma(1+\hat{\sigma})$ and type="lp" calculates $\boldsymbol{x}^{\prime} \hat{\boldsymbol{\beta}}$ only. We should not use them to predict MTTF and there are no software to calculate the minimum prediction error survival time

We use the Delta method to calculate the $95 \%$ confidence interval of the median survival time. The standard error of the predicted median time is calculated using $\mathrm{Eq}(22)$

$$
\begin{aligned}
& S E=\left\{\left(\begin{array}{c}
\frac{\partial E\left(\hat{t}_{i}\right)}{\partial \hat{\boldsymbol{\beta}}} \\
\frac{\partial E\left(t_{i}\right)}{\partial \hat{\sigma}}
\end{array}\right)^{t} \Sigma_{\hat{\sigma} \hat{\boldsymbol{\beta}}}\left(\begin{array}{c}
\frac{\partial E\left(\hat{t}_{i}\right)}{\partial \hat{\boldsymbol{\beta}}} \\
\frac{\partial E\left(t_{i}\right)}{\partial \hat{\sigma}}
\end{array}\right)\right\}^{\frac{1}{2}} \\
& =\left\{\left(\begin{array}{l}
\frac{\partial(\log 2)^{\hat{\sigma}} \exp \left(\boldsymbol{x}_{\boldsymbol{i}}^{\prime} \hat{\boldsymbol{\beta}}\right)}{\partial \hat{\boldsymbol{\beta}}} \\
\frac{\partial(\log 2)^{\hat{\sigma}} \exp \left(\boldsymbol{x}_{\boldsymbol{i}}^{\prime} \hat{\boldsymbol{\beta}}\right)}{\partial \hat{\sigma}}
\end{array}\right)^{t} \Sigma_{\hat{\sigma} \hat{\boldsymbol{\beta}}}\left(\begin{array}{c}
\frac{\partial(\log 2)^{\hat{\sigma}} \exp \left(\boldsymbol{x}_{\boldsymbol{i}}^{\prime} \hat{\boldsymbol{\beta}}\right)}{\partial \hat{\boldsymbol{\beta}}} \\
\frac{\partial(\log 2)^{\hat{\sigma}} \exp \left(\boldsymbol{x}_{\boldsymbol{i}}^{\prime} \hat{\boldsymbol{\beta}}\right)}{\partial \hat{\sigma}}
\end{array}\right)\right\}^{\frac{1}{2}} \\
& =\left\{\left(\begin{array}{l}
(\log 2)^{\hat{\sigma}} \exp \left(\boldsymbol{x}^{\prime} \hat{\boldsymbol{\beta}}\right) \\
(\log 2)^{\hat{\sigma}} \exp \left(\boldsymbol{x}^{\prime} \hat{\boldsymbol{\beta}}\right) * \text { stage } 2 \\
(\log 2)^{\hat{\sigma}} \exp \left(\boldsymbol{x}^{\prime} \hat{\boldsymbol{\beta}}\right) * \text { stage } 3 \\
(\log 2)^{\hat{\sigma}} \exp \left(\boldsymbol{x}^{\prime} \hat{\boldsymbol{\beta}}\right) * \text { stage } 4 \\
(\log 2)^{\hat{\sigma}} \exp \left(\boldsymbol{x}^{\prime} \hat{\boldsymbol{\beta}}\right) * \text { age } \\
(\log 2)^{\hat{\sigma}} \log (\log 2) \exp \left(\boldsymbol{x}^{\prime} \hat{\boldsymbol{\beta}}\right)
\end{array}\right)^{t} \Sigma_{\hat{\sigma} \hat{\boldsymbol{\beta}}}\left(\begin{array}{l}
(\log 2)^{\hat{\sigma}} \exp \left(\boldsymbol{x}^{\prime} \hat{\boldsymbol{\beta}}\right) \\
(\log 2)^{\hat{\sigma}} \exp \left(\boldsymbol{x}^{\prime} \hat{\boldsymbol{\beta}}\right) * \text { stage } 2 \\
(\log 2)^{\hat{\sigma}} \exp \left(\boldsymbol{x}^{\prime} \hat{\boldsymbol{\beta}}\right) * \text { stage } 3 \\
(\log 2)^{\hat{\sigma}} \exp \left(\boldsymbol{x}^{\prime} \hat{\boldsymbol{\beta}}\right) * \text { stage } 4 \\
(\log 2)^{\hat{\sigma}} \exp \left(\boldsymbol{x}^{\prime} \hat{\boldsymbol{\beta}}\right) * \text { age } \\
(\log 2)^{\hat{\sigma}} \log (\log 2) \exp \left(\boldsymbol{x}^{\prime} \hat{\boldsymbol{\beta}}\right)
\end{array}\right)\right\}^{\frac{1}{2}}
\end{aligned}
$$

First, we need to find the variance-covariance matrix $\Sigma_{\hat{\sigma} \hat{\boldsymbol{\beta}}}$, which can be calculated by the observed Fisher information of the Weibull AFT model (calculated by most software). Again in R:

wr\$var

We get

$\begin{array}{lrrrrrr} & \text { (Intercept) } & \text { stage2 } & \text { stage3 } & \text { stage4 } & \text { age } & \text { Log(scale) } \\ \text { (Intercept) } & 0.817 & -0.09049 & -0.08479 & -0.0444 & -0.01114 & 0.02591 \\ \text { stage2 } & -0.090 & 0.16611 & 0.05319 & 0.0507 & 0.00057 & 0.00016 \\ \text { stage3 } & -0.085 & 0.05319 & 0.10237 & 0.0567 & 0.00042 & -0.00731 \\ \text { stage4 } & -0.044 & 0.05068 & 0.05668 & 0.1320 & -0.00020 & -0.01070 \\ \text { age } & -0.011 & 0.00057 & 0.00042 & -0.0002 & 0.00016 & -0.00026 \\ \text { Log(scale) } & 0.026 & 0.00016 & -0.00731 & -0.0107 & -0.00026 & 0.01501\end{array}$

Note, $\mathrm{R}$ generates the variance-covariance of coefficients as $\log$ (scale), so we need to change the $\log$ (scale) to scale by performing extra calculations. Using formulas on page 401 of the book by John Klein [11], set $g_{1}(\sigma, \boldsymbol{\beta})=\boldsymbol{\beta}$ and $g_{2}(\sigma, \boldsymbol{\beta})=\sigma=e^{\log (\sigma)}$, i.e 
$\sigma=e^{\theta}$ is a function of $\theta$ and $\theta=\log (\sigma)$, we get

$$
\begin{aligned}
& \operatorname{Cov}\left(\beta_{0}, \sigma,\right)=\operatorname{Cov}\left(\beta_{0}, e^{\log (\sigma)}\right)=\operatorname{Cov}\left(\beta_{0}, \log (\sigma)\right) * \sigma=0.02292735 \\
& \operatorname{Cov}\left(\beta_{1}, \sigma,\right)=\operatorname{Cov}\left(\beta_{1}, e^{\log (\sigma)}\right)=\operatorname{Cov}\left(\beta_{1}, \log (\sigma)\right) * \sigma=0.0001403178 \\
& \operatorname{Cov}\left(\beta_{2}, \sigma,\right)=\operatorname{Cov}\left(\beta_{2}, e^{\log (\sigma)}\right)=\operatorname{Cov}\left(\beta_{2}, \log (\sigma)\right) * \sigma-0.006469443 \\
& \operatorname{Cov}\left(\beta_{3}, \sigma,\right)=\operatorname{Cov}\left(\beta_{3}, e^{\log (\sigma)}\right)=\operatorname{Cov}\left(\beta_{3}, \log (\sigma)\right) * \sigma=-0.009470604 \\
& \operatorname{Cov}\left(\beta_{4}, \sigma,\right)=\operatorname{Cov}\left(\beta_{4}, e^{\log (\sigma)}\right)=\operatorname{Cov}\left(\beta_{4}, \log (\sigma)\right) * \sigma=-0.0002297781 \\
& \operatorname{Cov}(\sigma)=\operatorname{Cov}\left(e^{\log (\sigma)}\right)=\left(e^{\log (\sigma)}\right)^{2} \operatorname{Cov}(\log (\sigma))=\sigma^{2} \operatorname{Var}(\log (\sigma))=0.0117501
\end{aligned}
$$

Now we will use these six values to replace the last row and column of the variance-covariance matrix of the coefficients and $\log ($ scale). We get

$$
[, 1] \quad[, 2] \quad[, 3] \quad[, 4] \quad[, 5] \quad[, 6]
$$

$[1] \quad 0.817-0.09049-0.08479-0.0444-,0.01114 \quad 0.02293$

$\left[\begin{array}{lllllll}{[2,]} & -0.090 & 0.16611 & 0.05319 & 0.0507 & 0.00057 & 0.00014\end{array}\right.$

$\left[\begin{array}{lllllll}{[3,]} & -0.085 & 0.05319 & 0.10237 & 0.0567 & 0.00042 & -0.00647\end{array}\right.$

$\left[\begin{array}{lllllll}{[4,]} & -0.044 & 0.05068 & 0.05668 & 0.1320 & -0.00020 & -0.00947\end{array}\right.$

$\left[\begin{array}{lllllll}5,] & -0.011 & 0.00057 & 0.00042 & -0.0002 & 0.00016 & -0.00023\end{array}\right.$

$\begin{array}{lllllllll}{[6,]} & 0.023 & 0.00014 & -0.00647 & -0.0095 & -0.00023 & 0.01175\end{array}$

This is the $\Sigma_{\hat{\sigma} \hat{\boldsymbol{\beta}}}$ matrix we needed.

If we use SAS software, we can directly get the variance-covariance matrix of $\hat{\boldsymbol{\beta}}$ and $\hat{\sigma}$ by using the following statements

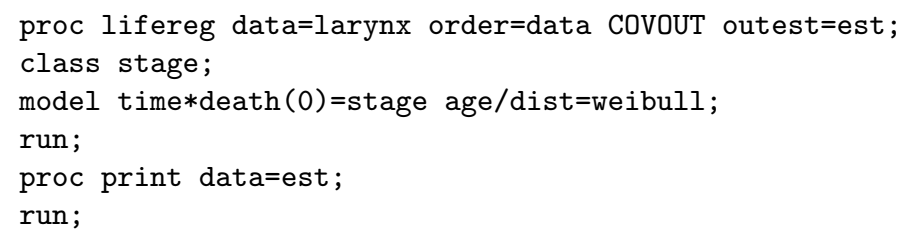

The right side vector of the $\mathrm{Eq}(27)$ is calculated as

$$
\begin{aligned}
& \left(\begin{array}{l}
(\log 2)^{\hat{\sigma}} \exp \left(\boldsymbol{x}^{\prime} \hat{\boldsymbol{\beta}}\right) \\
(\log 2)^{\hat{\sigma}} \exp \left(\boldsymbol{x}^{\prime} \hat{\boldsymbol{\beta}}\right) * \text { stage } 2 \\
(\log 2)^{\hat{\sigma}} \exp \left(\boldsymbol{x}^{\prime} \hat{\boldsymbol{\beta}}\right) * \text { stage } \\
(\log 2)^{\hat{\sigma}} \exp \left(\boldsymbol{x}^{\prime} \hat{\boldsymbol{\beta}}\right) * \text { stage } 4 \\
(\log 2)^{\hat{\sigma}} \exp \left(\boldsymbol{x}^{\prime} \hat{\boldsymbol{\beta}}\right) * \text { age } \\
(\log 2)^{\hat{\sigma}} \log (\log 2) \exp \left(\boldsymbol{x}^{\prime} \hat{\boldsymbol{\beta}}\right)
\end{array}\right) \\
& =\left(\begin{array}{c}
(\log 2)^{0.885} \exp (3.5288-0.1477 * 1-0.5866 * 0-1.5441 * 0-0.0175 * 74) \\
(\log 2)^{0.885} \exp (3.5288-0.1477 * 1-0.5866 * 0-1.5441 * 0-0.0175 * 74) * 1 \\
(\log 2)^{0.885} \exp (3.5288-0.1477 * 1-0.5866 * 0-1.5441 * 0-0.0175 * 74) * 0 \\
(\log 2)^{0.885} a \exp (3.5288-0.1477 * 1-0.5866 * 0-1.5441 * 0-0.0175 * 74) * 0 \\
(\log 2)^{0.885} \exp (3.5288-0.1477 * 1-0.5866 * 0-1.5441 * 0-0.0175 * 74) * 74 \\
(\log 2)^{0.885} \log \log (\log 2) \exp (3.5288-0.1477 * 1-0.5866 * 0-1.5441 * 0-0.0175 * 74)
\end{array}\right) \\
& =\left(\begin{array}{c}
5.8383 \\
5.8383 \\
0 \\
0 \\
432.03 \\
-7.7915
\end{array}\right)
\end{aligned}
$$


Now we have everything to calculate the standard error of the median survival time.

$$
\begin{aligned}
S E & =\left\{\left(\begin{array}{c}
5.8383 \\
5.8383 \\
0 \\
0 \\
432.03 \\
-7.7915
\end{array}\right)^{t}\left(\begin{array}{cccccc}
0.817 & -0.0905 & -0.0848 & -0.0444 & -0.0111 & 0.0229 \\
-0.090 & 0.1661 & 0.0532 & 0.0507 & 0.00057 & 0.00014 \\
-0.0859 & 0.0532 & 0.1024 & 0.0567 & 0.00042 & -0.0065 \\
-0.044 & 0.0507 & 0.0567 & 0.1320 & -0.00020 & -0.0095 \\
-0.011 & 0.00057 & 0.0004 & -0.00020 & 0.00016 & -0.00023 \\
0.023 & 0.00014 & -0.0065 & -0.0095 & -0.00023 & 0.01175
\end{array}\right)\left(\begin{array}{c}
5.8383 \\
5.8383 \\
0 \\
0 \\
432.03 \\
-7.7915
\end{array}\right)\right\}^{\frac{1}{2}} \\
& =2.156133
\end{aligned}
$$

Therefore, the $95 \%$ confidence interval is given as

$95 \% C I:\left(5.83-1.96 * 2.16<\right.$ Median $\left._{46}<5.83+1.96 * 2.16\right)=(1.60$ to 10.01$)$ months.

This result indicates we are $95 \%$ confident that the survival time will be within 1.60 to 10.01 months. We can also use the $\mathrm{R}$ build in function predict to predict the median survival time.

Median46<-predict (wr, newdata=data. frame (stage=2, age=74), type="quantile" , $\mathrm{p}=0.5$, se.fit=TRUE)

Median46

We get

\$fit

5.838288

\$se.fit

2.095133

This standard error differs slightly to our calculations as R uses Greedwood's formula to calculate the standard error of the survival function. [11]

\section{Assess the point prediction accuracy}

Two methods of assessing accuracy of predicted survival time have been proposed by Parkes and Christakis and Lamont. [12,13] Parkes suggests let $t$ be the observed survival time and $p$ be the predicted time. If $p / k \leq t \leq k p$ then the point prediction $p$ is defined as "accurate" and any value outside the interval is "inaccurate". Parkes proposes $k=2$ as suitable. As a more strict assessment, Christakis and Lamont purposed a 33\% rule to measure accuracy, where the observed time is divided by the predicted survival time and defined as "accurate" if this quotient lies between 0.67 and 1.33. Values less than 0.67 or greater than 1.33 are defined as "errors". We compared the accuracy of our example using Parkes method $(k=2)$ and Christakis' method. The accuracy rate (i.e. proportion of "accurate" prediction over the total sample size) is presented in Table 1.

\section{Discussion}

In this paper, we introduced how to use a Weibull AFT model to predict when a health-related event will happen. Expected survival time, median survival time and minimum prediction error survival time from baseline to event were estimated and prediction accuracy assessed using Parkes' and Christakis and Lamont's method. When we fixed $k=2$ the accuracy was $55.6 \%$ for median, $50 \%$ for MTTF and $51.1 \%$ for MPET. When used the method as suggested by Christakis and Lamont, the accuracy rate decreased to $30.0 \%, 37.8 \%$ and $33.3 \%$ (data not shown), respectively. Our example is limited as the sample is small and we only used two predictors. However, with a larger sample size and more predictors, accuracy may improve. In this example, we did not observe minimum prediction error time to be more accurate than median survival time.
192

91 
Parametric survival models are advantageous in predicting survival time compared to semi-parametric Cox regression models. The Cox regression model which can be specified as $S_{i}\left(t \mid x_{i}\right)=S_{0}(t)^{\exp \left(\boldsymbol{x}_{\boldsymbol{i}}^{\prime} \boldsymbol{\beta}\right)}$ cannot predict time directly but calculates the probability of an event occurring within a specified timeframe. However, one disadvantage of parametric survival models such as Weibull AFT is the need to to make stronger assumptions than semi-parametric models. [14]

Currently, most clinical prediction models describe a patient's likelihood of having or developing a certain disease as a traditional probability value or a risk score that is based on calculated probability. [15] However, probabilities are not intuitive to the general population and probability itself can be defined in different ways. [16] In practice, the time axis remains the most natural measure for both clinicians and patients. It is much easier to understand a survival time rather than probability of survival in a certain timepoint. Predicting when an event will happen provides a practical and interpretable guide for clinicians, health care providers and patients and and can help with decision making over remaining lifespan.

Upon recognizing that the Weibull AFT can be adapted from an engineering reliability framework to a medical framework, the next step involves developing a real prediction tool for medical predictive purposes, applying models to larger datasets and performing rigorous internal and external validation procedures. Such steps are outlined in the book Clinical prediction models: a practical approach to development, validation, and updating for the steps to develop a real prediction tool. [17]

\section{References}

1. Sullivan LM, Massaro JM, D'Agostino Sr RB. Presentation of multivariate data for clinical use: The Framingham Study risk score functions. Statistics in medicine 2004;23:1631-60.

2. Kanis J, McCloskey E, Johansson H, et al. Development and use of FRAXß in osteoporosis. Osteoporosis international 2010;21:407-13.

3. Ali JB, Chebel-Morello B, Saidi L, et al. Accurate bearing remaining useful life prediction based on Weibull distribution and artificial neural network. Mechanical Systems and Signal Processing 2015;56:150-72.

4. Fu B, Labuza TP. Shelf-life prediction: theory and application. Food Control 1993;4:125-33.

5. Li X, Lu WF, Zhai L, et al. Remaining life prediction of cores based on data-driven and physical modeling methods. Handbook of Manufacturing Engineering and Technology. Springer; 2015. p. 3239-64.

6. Gorgoso-Varela JJ, Rojo-Alboreca A. Use of Gumbel and Weibull functions to model extreme values of diameter distributions in forest stands. Annals of forest science. 2014;71(7):741-50.

7. Lai C-D. Generalized Weibull Distributions. Generalized Weibull Distributions: Springer; 2014. p. 23-75.

8. Ho L, Silva A. Unbiased estimators for mean time to failure and percentiles in a Weibull regression model. International Journal of Quality \& Reliability Management.2006;23(3):323-39.

9. Henderson R, Jones M, Stare J. Accuracy of point predictions in survival analysis. Statistics in medicine. 2001;20(20):3083-96. 
10. Klein JP, Moeschberger ML. Survival analysis: techniques for censored and truncated data. Springer Science \& Business Media; 2005.

11. Collett D. Modelling survival data in medical research Chapman and Hall/CRC; 2015 .

12. Parkes CM. Accuracy of predictions of survival in later stages of cancer. British medical journal. 1972;2(5804):29.

13. Christakis NA, Smith JL, Parkes CM, Lamont EB. Extent and determinants of error in doctors' prognoses in terminally ill patients: prospective cohort studyCommentary: Why do doctors overestimate? Commentary: Prognoses should be based on proved indices not intuition. Bmj. 2000;320(7233):469-73.

14. Nardi A, Schemper M. Comparing Cox and parametric models in clinical studies. Statistics in medicine. 2003;22(23):3597-610.

15. Lee Y-h, Bang H, Kim DJ. How to establish clinical prediction models. Endocrinology and Metabolism. 2016;31(1):38-44.

16. Saunders S. What is probability? Quo vadis quantum mechanics? Springer; 2005. p. 209-38.

17. Steyerberg EW. Clinical prediction models: a practical approach to development, validation, and updating. Springer Science \& Business Media; 2008. 
Table 1. Prediction results and accuracy (last digit in predicted time: 0 inaccurate, 1 , accurate)

\begin{tabular}{|c|c|c|c|c|c|c|c|}
\hline ID & stage & age & death & time & Median(95\% CI) & MTTF & MPET \\
\hline 1 & 1 & 77 & 1 & 0.6 & $6.42(3.16,9.68), 0$ & $8.47,0$ & $8.11,0$ \\
\hline 2 & 1 & 53 & 1 & 1.3 & $9.77(4.07,15.46), 0$ & $12.9,0$ & $12.34,0$ \\
\hline 3 & 1 & 45 & 1 & 2.4 & $11.23(3.07,19.39), 0$ & $14.84,0$ & $14.19,0$ \\
\hline 4 & 1 & 57 & 0 & 2.5 & $9.11(4.32,13.89), 0$ & $12.03,0$ & $11.5,0$ \\
\hline 5 & 1 & 58 & 1 & 3.2 & $8.95(4.36,13.54), 0$ & $11.82,0$ & $11.3,0$ \\
\hline 6 & 1 & 51 & 0 & 3.2 & $10.11(3.88,16.34), 0$ & $13.36,0$ & $12.78,0$ \\
\hline 7 & 1 & 76 & 1 & 3.3 & $6.54(3.29,9.78), 1$ & $8.62,0$ & $8.25,0$ \\
\hline 8 & 1 & 63 & 0 & 3.3 & $8.2(4.37,12.03), 0$ & $10.83,0$ & $10.36,0$ \\
\hline 9 & 1 & 43 & 1 & 3.5 & $11.63(2.72,20.54), 0$ & $15.36,0$ & $14.7,0$ \\
\hline 10 & 1 & 60 & 1 & 3.5 & $8.64(4.4,12.89), 0$ & $11.41,0$ & $10.91,0$ \\
\hline 11 & 1 & 52 & 1 & 4 & $9.94(3.98,15.89), 0$ & $13.13,0$ & $12.55,0$ \\
\hline 12 & 1 & 63 & 1 & 4 & $8.2(4.37,12.03), 0$ & $10.83,0$ & $10.36,0$ \\
\hline 13 & 1 & 86 & 1 & 4.3 & $5.49(1.96,9.01), 1$ & $7.24,1$ & $6.92,1$ \\
\hline 14 & 1 & 48 & 0 & 4.5 & $10.66(3.52,17.79), 0$ & $14.08,0$ & $13.47,0$ \\
\hline 15 & 1 & 68 & 0 & 4.5 & $7.52(4.12,10.91), 1$ & $9.92,0$ & $9.49,0$ \\
\hline 16 & 1 & 81 & 1 & 5.3 & $5.99(2.63,9.34), 1$ & $7.9,1$ & $7.56,1$ \\
\hline 17 & 1 & 70 & 0 & 5.5 & $7.26(3.96,10.56), 1$ & $9.58,1$ & $9.16,1$ \\
\hline 18 & 1 & 58 & 0 & 5.9 & $8.95(4.36,13.54), 1$ & $11.82,0$ & $11.3,1$ \\
\hline 19 & 1 & 47 & 0 & 5.9 & $10.84(3.38,18.31), 1$ & $14.33,0$ & $13.7,0$ \\
\hline 20 & 1 & 75 & 1 & 6 & $6.65(3.41,9.89), 1$ & $8.78,1$ & $8.39,1$ \\
\hline 21 & 1 & 77 & 0 & 6.1 & $6.42(3.16,9.68), 1$ & $8.47,1$ & $8.11,1$ \\
\hline 22 & 1 & 64 & 0 & 6.2 & $8.06(4.34,11.77), 1$ & $10.64,1$ & $10.18,1$ \\
\hline 23 & 1 & 77 & 1 & 6.4 & $6.42(3.16,9.68), 1$ & $8.47,1$ & $8.11,1$ \\
\hline 24 & 1 & 67 & 1 & 6.5 & $7.65(4.19,11.1), 1$ & $10.1,1$ & $9.66,1$ \\
\hline 25 & 1 & 79 & 0 & 6.5 & $6.2(2.9,9.5), 1$ & $8.18,1$ & $7.83,1$ \\
\hline 26 & 1 & 61 & 0 & 6.7 & $8.49(4.4,12.58), 1$ & $11.21,1$ & $10.73,1$ \\
\hline 27 & 1 & 66 & 0 & 7 & $7.78(4.25,11.31), 1$ & $10.27,1$ & $9.83,1$ \\
\hline 28 & 1 & 68 & 1 & 7.4 & $7.52(4.12,10.91), 1$ & $9.92,1$ & $9.49,1$ \\
\hline 29 & 1 & 73 & 0 & 7.4 & $6.89(3.65,10.12), 1$ & $9.09,1$ & $8.69,1$ \\
\hline 30 & 1 & 56 & 0 & 8.1 & $9.27(4.28,14.26), 1$ & $12.24,1$ & $11.71,1$ \\
\hline 31 & 1 & 73 & 0 & 8.1 & $6.89(3.65,10.12), 1$ & $9.09,1$ & $8.69,1$ \\
\hline 32 & 1 & 58 & 0 & 9.6 & $8.95(4.36,13.54), 1$ & $11.82,1$ & $11.3,1$ \\
\hline 33 & 1 & 68 & 0 & 10.7 & $7.52(4.12,10.91), 1$ & $9.92,1$ & $9.49,1$ \\
\hline 34 & 2 & 86 & 1 & 0.2 & $4.73(0.68,8.78), 0$ & $6.25,0$ & $5.97,0$ \\
\hline 35 & 2 & 64 & 1 & 1.8 & $6.95(2.37,11.54), 0$ & $9.18,0$ & $8.78,0$ \\
\hline 36 & 2 & 63 & 1 & 2 & $7.07(2.4,11.75), 0$ & $9.34,0$ & $8.93,0$ \\
\hline 37 & 2 & 71 & 0 & 2.2 & $6.15(1.96,10.34), 0$ & $8.12,0$ & $7.77,0$ \\
\hline 38 & 2 & 67 & 0 & 2.6 & $6.6(2.22,10.97), 0$ & $8.71,0$ & $8.33,0$ \\
\hline 39 & 2 & 51 & 0 & 3.3 & $8.72(2.28,15.17), 0$ & $11.52,0$ & $11.02,0$ \\
\hline 40 & 2 & 70 & 1 & 3.6 & $6.26(2.03,10.49), 1$ & $8.26,0$ & $7.9,0$ \\
\hline 41 & 2 & 72 & 0 & 3.6 & $6.05(1.89,10.2), 1$ & $7.98,0$ & $7.63,0$ \\
\hline 42 & 2 & 81 & 1 & 4 & $5.17(1.13,9.21), 1$ & $6.82,1$ & $6.52,1$ \\
\hline 43 & 2 & 47 & 0 & 4.3 & $9.36(1.98,16.73), 0$ & $12.36,0$ & $11.82,0$ \\
\hline 44 & 2 & 64 & 0 & 4.3 & $6.95(2.37,11.54), 1$ & $9.18,0$ & $8.78,0$ \\
\hline 45 & 2 & 66 & 0 & 5 & $6.71(2.28,11.15), 1$ & $8.86,1$ & $8.48,1$ \\
\hline
\end{tabular}


bioRxiv preprint doi: https://doi.org/10.1101/362186; this version posted August 27, 2018. The copyright holder for this preprint (which was not certified by peer review) is the author/funder, who has granted bioRxiv a license to display the preprint in perpetuity. It is made available under aCC-BY 4.0 International license.

Table 1 continued Prediction results and accuracy (last digit in predicted time: 0 inaccurate, 1 , accurate)

\begin{tabular}{|c|c|c|c|c|c|c|c|}
\hline ID & stage & age & death & time & Median $(95 \%$ CI $)$ & MTTF & MPET \\
\hline 46 & 2 & 74 & 1 & 6.2 & $5.84(1.73,9.94), 1$ & $7.7,1$ & $7.37,1$ \\
\hline 47 & 2 & 62 & 1 & 7 & $7.2(2.43,11.97), 1$ & $9.51,1$ & $9.09,1$ \\
\hline 48 & 2 & 50 & 0 & 7.5 & $8.88(2.22,15.54), 1$ & $11.73,1$ & $11.22,1$ \\
\hline 49 & 2 & 53 & 0 & 7.6 & $8.42(2.38,14.47), 1$ & $11.13,1$ & $10.64,1$ \\
\hline 50 & 2 & 61 & 0 & 9.3 & $7.33(2.46,12.2), 1$ & $9.67,1$ & $9.25,1$ \\
\hline 51 & 3 & 49 & 1 & 0.3 & $5.83(2.41,9.24), 0$ & $7.69,0$ & $7.36,0$ \\
\hline 52 & 3 & 71 & 1 & 0.3 & $3.97(2.19,5.75), 0$ & $5.24,0$ & $5.01,0$ \\
\hline 53 & 3 & 57 & 1 & 0.5 & $5.07(2.68,7.45), 0$ & $6.69,0$ & $6.4,0$ \\
\hline 54 & 3 & 79 & 1 & 0.7 & $3.45(1.56,5.34), 0$ & $4.55,0$ & $4.35,0$ \\
\hline 55 & 3 & 82 & 1 & 0.8 & $3.27(1.32,5.23), 0$ & $4.32,0$ & $4.13,0$ \\
\hline 56 & 3 & 49 & 1 & 1 & $5.83(2.41,9.24), 0$ & $7.69,0$ & $7.36,0$ \\
\hline 57 & 3 & 60 & 1 & 1.3 & $4.81(2.68,6.94), 0$ & $6.35,0$ & $6.07,0$ \\
\hline 58 & 3 & 64 & 1 & 1.6 & $4.48(2.58,6.39), 0$ & $5.92,0$ & $5.66,0$ \\
\hline 59 & 3 & 74 & 1 & 1.8 & $3.76(1.96,5.56), 0$ & $4.97,0$ & $4.75,0$ \\
\hline 60 & 3 & 72 & 1 & 1.9 & $3.9(2.12,5.68), 0$ & $5.14,0$ & $4.92,0$ \\
\hline 61 & 3 & 53 & 1 & 1.9 & $5.43(2.6,8.27), 0$ & $7.17,0$ & $6.86,0$ \\
\hline 62 & 3 & 54 & 1 & 3.2 & $5.34(2.63,8.05), 1$ & $7.05,0$ & $6.74,0$ \\
\hline 63 & 3 & 81 & 1 & 3.5 & $3.33(1.4,5.27), 1$ & $4.39,1$ & $4.2,1$ \\
\hline 64 & 3 & 52 & 0 & 3.7 & $5.53(2.56,8.49), 1$ & $7.3,1$ & $6.98,1$ \\
\hline 65 & 3 & 66 & 0 & 4.5 & $4.33(2.49,6.17), 1$ & $5.71,1$ & $5.47,1$ \\
\hline 66 & 3 & 54 & 0 & 4.8 & $5.34(2.63,8.05), 1$ & $7.05,1$ & $6.74,1$ \\
\hline 67 & 3 & 63 & 0 & 4.8 & $4.56(2.61,6.51), 1$ & $6.02,1$ & $5.76,1$ \\
\hline 68 & 3 & 59 & 1 & 5 & $4.89(2.69,7.1), 1$ & $6.46,1$ & $6.18,1$ \\
\hline 69 & 3 & 49 & 0 & 5 & $5.83(2.41,9.24), 1$ & $7.69,1$ & $7.36,1$ \\
\hline 70 & 3 & 69 & 0 & 5.1 & $4.11(2.32,5.89), 1$ & $5.42,1$ & $5.19,1$ \\
\hline 71 & 3 & 70 & 1 & 6.3 & $4.04(2.26,5.82), 1$ & $5.33,1$ & $5.1,1$ \\
\hline 72 & 3 & 65 & 1 & 6.4 & $4.41(2.54,6.27), 1$ & $5.82,1$ & $5.56,1$ \\
\hline 73 & 3 & 65 & 0 & 6.5 & $4.41(2.54,6.27), 1$ & $5.82,1$ & $5.56,1$ \\
\hline 74 & 3 & 68 & 1 & 7.8 & $4.18(2.38,5.98), 1$ & $5.52,1$ & $5.28,1$ \\
\hline 75 & 3 & 78 & 0 & 8 & $3.51(1.64,5.38), 0$ & $4.63,1$ & $4.43,1$ \\
\hline 76 & 3 & 69 & 0 & 9.3 & $4.11(2.32,5.89), 0$ & $5.42,1$ & $5.19,1$ \\
\hline 77 & 3 & 51 & 0 & 10.1 & $5.63(2.52,8.73), 1$ & $7.43,1$ & $7.11,1$ \\
\hline 78 & 4 & 65 & 1 & 0.1 & $1.69(0.77,2.61), 0$ & $2.23,0$ & $2.14,0$ \\
\hline 79 & 4 & 71 & 1 & 0.3 & $1.52(0.7,2.34), 0$ & $2.01,0$ & $1.92,0$ \\
\hline 80 & 4 & 76 & 1 & 0.4 & $1.4(0.61,2.18), 0$ & $1.84,0$ & $1.76,0$ \\
\hline 81 & 4 & 65 & 1 & 0.8 & $1.69(0.77,2.61), 0$ & $2.23,0$ & $2.14,0$ \\
\hline 82 & 4 & 78 & 1 & 0.8 & $1.35(0.56,2.13), 1$ & $1.78,0$ & $1.7,0$ \\
\hline 83 & 4 & 41 & 1 & 1 & $2.57(0.3,4.84), 0$ & $3.4,0$ & $3.25,0$ \\
\hline 84 & 4 & 68 & 1 & 1.5 & $1.6(0.74,2.47), 1$ & $2.12,1$ & $2.03,1$ \\
\hline 85 & 4 & 69 & 1 & 2 & $1.58(0.73,2.42), 1$ & $2.08,1$ & $1.99,1$ \\
\hline 86 & 4 & 62 & 1 & 2.3 & $1.78(0.78,2.79), 1$ & $2.35,1$ & $2.25,1$ \\
\hline 87 & 4 & 74 & 0 & 2.9 & $1.44(0.65,2.24), 0$ & $1.91,1$ & $1.82,1$ \\
\hline 88 & 4 & 71 & 1 & 3.6 & $1.52(0.7,2.34), 0$ & $2.01,1$ & $1.92,1$ \\
\hline 89 & 4 & 84 & 1 & 3.8 & $1.21(0.42,2.01), 0$ & $1.6,0$ & $1.53,0$ \\
\hline 90 & 4 & 48 & 0 & 4.3 & $2.28(0.57,3.99), 1$ & $3.01,1$ & $2.87,1$ \\
\hline Accuracy rate(\%) & & & & & $55.6 \%(50 / 90)$ & $50 \%(45 / 90)$ & $51.1 \%(46 / 90)$ \\
\hline
\end{tabular}

